\title{
Evaluation of Liver Fibrosis Using Texture Analysis on Combined-Contrast-Enhanced Magnetic Resonance Images at 3.0T
}

\author{
Takeshi Yokoo, ${ }^{1,2}$ Tanya Wolfson, ${ }^{3}$ Keiko Iwaisako, ${ }^{1,4,5}$ Michael R. Peterson, ${ }^{6}$ \\ Haresh Mani, ${ }^{7}$ Zachary Goodman, ${ }^{8}$ Christopher Changchien, ${ }^{1}$ \\ Michael S. Middleton, ${ }^{1}$ Anthony C. Gamst, ${ }^{3}$ Sameer M. Mazhar, ${ }^{4}$ Yuko Kono, ${ }^{1}$ \\ Samuel B. Ho, ${ }^{4,9}$ and Claude B. Sirlin ${ }^{1}$ \\ ${ }^{1}$ Departments of Radiology, University of California, San Diego, CA 92103, USA \\ ${ }^{2}$ Department of Radiology and Advanced Imaging Research Center, University of Texas Southwestern Medical Center, \\ 2201 Inwood Road, NE2.210B, Dallas, TX 75390-9085, USA \\ ${ }^{3}$ Computational and Applied Statistics Laboratory, San Diego Supercomputer Center, University of California, \\ San Diego, CA 92093, USA \\ ${ }^{4}$ Departments of Medicine, University of California, San Diego, CA 92103, USA \\ ${ }^{5}$ Department of Target Therapy Oncology, Kyoto University Graduate School of Medicine, Kyoto, Japan \\ ${ }^{6}$ Departments of Pathology, University of California, San Diego, CA 92103, USA \\ ${ }^{7}$ Department of Pathology, Penn State Hershey Medical Center, Hershey, PA 17033, USA \\ ${ }^{8}$ Center for Liver Diseases, Inova Fairfax Hospital, Falls Church, VA 22042, USA \\ ${ }^{9}$ VA San Diego Healthcare System, San Diego, CA 92161, USA
}

Correspondence should be addressed to Takeshi Yokoo; takeshi.yokoo@utsouthwestern.edu

Received 29 July 2014; Revised 15 October 2014; Accepted 18 October 2014

Academic Editor: Trevor Andrews

Copyright (C) 2015 Takeshi Yokoo et al. This is an open access article distributed under the Creative Commons Attribution License, which permits unrestricted use, distribution, and reproduction in any medium, provided the original work is properly cited.

\begin{abstract}
Purpose. To noninvasively assess liver fibrosis using combined-contrast-enhanced (CCE) magnetic resonance imaging (MRI) and texture analysis. Materials and Methods. In this IRB-approved, HIPAA-compliant prospective study, 46 adults with newly diagnosed HCV infection and recent liver biopsy underwent CCE liver MRI following intravenous administration of superparamagnetic iron oxides (ferumoxides) and gadolinium DTPA (gadopentetate dimeglumine). The image texture of the liver was quantified in regions-of-interest by calculating 165 texture features. Liver biopsy specimens were stained with Masson trichrome and assessed qualitatively (METAVIR fibrosis score) and quantitatively (\% collagen stained area). Using $L_{1}$ regularization path algorithm, two texture-based multivariate linear models were constructed, one for quantitative and the other for quantitative histology prediction. The prediction performance of each model was assessed using receiver operating characteristics (ROC) and correlation analyses. Results. The texture-based predicted fibrosis score significantly correlated with qualitative $(r=0.698, P<0.001)$ and quantitative $(r=0.757, P<0.001)$ histology. The prediction model for qualitative histology had $0.814-0.976$ areas under the curve (AUC), 0.659-1.000 sensitivity, 0.778-0.930 specificity, and 0.674-0.935 accuracy, depending on the binary classification threshold. The prediction model for quantitative histology had 0.742-0.950 AUC, 0.688-1.000 sensitivity, 0.679-0.857 specificity, and 0.696-0.848 accuracy, depending on the binary classification threshold. Conclusion. CCE MRI and texture analysis may permit noninvasive assessment of liver fibrosis.
\end{abstract}

\section{Introduction}

The ongoing epidemic of Chronic Liver Disease (CLD) is a major contributor to liver-related mortality and morbidity in the United States. More than 20,000 Americans die from CLD complications each year [1]. The most common etiologies for CLD are chronic hepatitis $\mathrm{C}$ virus (HCV) infection and alcoholic hepatitis $[2,3]$. Over 4 million Americans are HCV 

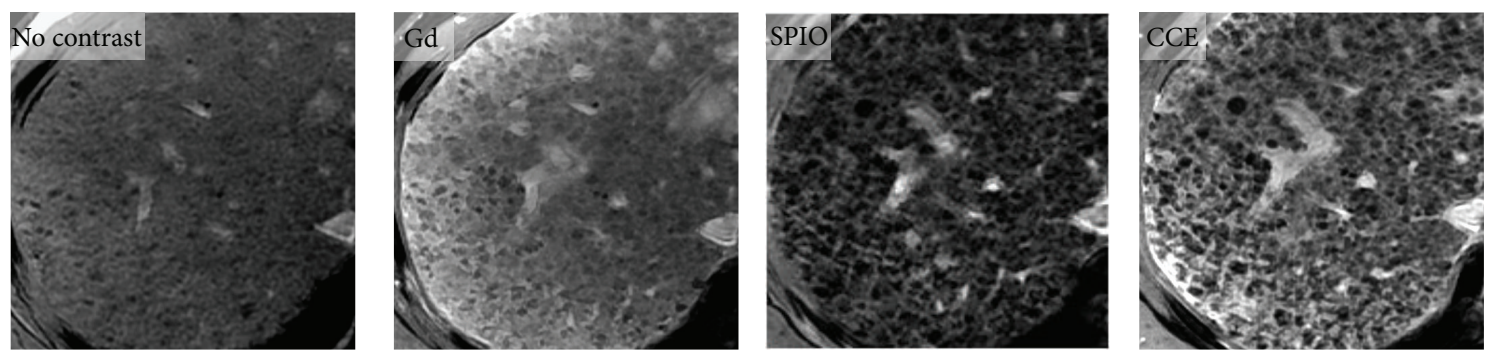

FIGURE 1: MR images of liver in 60-year old man with HCV-related cirrhosis. Noncontrast, Gd-only, SPIO-only, and CCE 2D breath-hold T1-weighted gradient-echo images of cirrhotic liver due to HCV. Abnormal reticular pattern of the liver parenchyma is better visualized on single-contrast-enhanced (Gd or SPIO) images than on unenhanced image and better visualized on CCE images than on single-contrastenhanced images. Gd: gadolinium; SPIO: superparamagnetic iron oxide, and CCE: combined contrast enhanced.
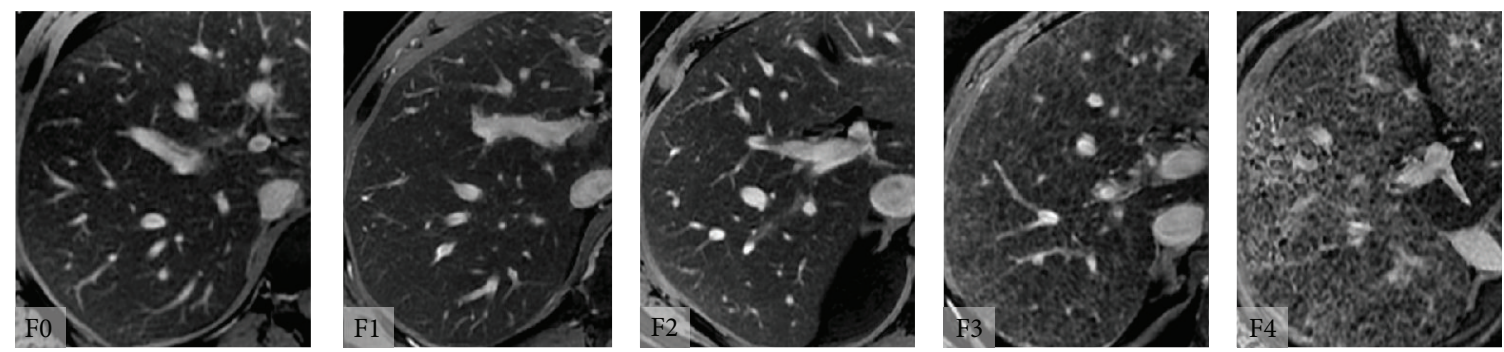

FIGURE 2: Combined contrast enhanced (CCE) MR images at various stages of fibrosis. CCE MR images in adults with chronic HCV infection and histologically determined Metavir fibrosis stages F0, F1, F2, F3, and F4. Subjectively, the reticular texture of the liver parenchyma becomes progressively more pronounced with increasing Metavir fibrosis stage.

carriers, but many are asymptomatic and unaware of their infection [4].

The common pathway in the natural history of CLD, including chronic $\mathrm{HCV}$ infection, is progressive liver fibrosis and ultimately cirrhosis [5]. Fibrosis indicates cumulative liver damage, contributes to the development of portal hypertension and hepatic dysfunction, and predicts poor clinical outcome $[6,7]$. Most liver-related mortality and morbidity occur in the cirrhotic population [8]. Assessment of liver fibrosis is therefore critical in the management of patients with CLD.

The current gold standard for fibrosis evaluation is liver biopsy. The severity of fibrosis due to HCV infection is often classified using an ordinal scale such as the Metavir system [9]. However, biopsy is invasive and thus problematic for frequent monitoring. Moreover, its interpretation is subjective, leading to inter- and intraobserver variability [10-12]. For these reasons, noninvasive and objective techniques are under investigation, including fibrosis-specific serum markers $[13,14]$, ultrasound elastography $[15,16]$, magnetic resonance (MR) elastography [17-19], diffusion weighted MR imaging [20-22], and single-contrast-enhanced MR imaging [23-25].

Another promising MR imaging-based technique is combined-contrast-enhanced (CCE) MR imaging [26]. This technique exploits the complementary effects of positive contrast enhancement by gadolinium-chelates (Gd) and negative enhancement by superparamagnetic iron oxide (SPIO) agents. Compared to noncontrast, Gd-enhanced, or SPIOenhanced images, CCE images better depict the reticular signal abnormalities associated with fibrosis as shown in Figure 1 [27]. The conspicuity of this pattern appears to parallel histologic fibrosis severity (Figure 2) suggesting that liver fibrosis can be assessed by the severity of the "texture" abnormality.

The potential role of texture analysis in liver fibrosis assessment was previously explored in retrospective studies using qualitative [26] and quantitative [26, 28-30] texture analysis. The purpose of this prospective study was to provide proof-of-concept that quantitative texture analysis using CCE MR imaging may permit noninvasively assess liver fibrosis in adults with $\mathrm{HCV}$ infection using CCE MR imaging.

\section{Method and Materials}

2.1. Study Design and Subjects. This prospective, crosssectional, observational clinical study was approved by an institutional review board and is HIPAA-compliant. Potential eligible subjects were referred for research MR imaging examination from hepatology clinics at our institution. Written informed consents were obtained. Selection criteria are listed in Table 1. Patient recruitment was stratified according to the fibrosis severity at liver biopsy and continued until at least five subjects in each fibrosis severity category (per clinical biopsy reports) were enrolled.

2.2. Liver Biopsy. Subjects had a percutaneous 16-gauge needle-core biopsy of the right hepatic lobe for clinical care by the referring hepatologists. Specimens were processed in 
TABLE 1: Selection criteria.

\begin{tabular}{ll}
\hline Inclusion criteria & Exclusion criteria \\
\hline $\begin{array}{l}\text { (i) Age }>18 \text { years } \\
\text { (ii) Newly diagnosed HCV infection, without clinically }\end{array}$ & (i) Estimated GFR $<60 \mathrm{~mL} / \mathrm{mL}(N=0$ potential subjects) \\
$\begin{array}{l}\text { overt cirrhosis } \\
\text { (iii) Recent or planned biopsy }{ }^{1}\end{array}$ & $\begin{array}{l}\text { (iii) Nondiagnostic biopsy or trichrome slide unavailable }(N=2) \\
\text { (iv) Contraindication to MR exam }(N=1)^{2}\end{array}$ \\
$\begin{array}{ll}\text { (iv) Willing and able to undergo CCE MRI exam within } \\
30 \text { days of biopsy }\end{array}$ & $\begin{array}{l}\text { (v) Lack of intravenous access }(N=1) \\
\text { (v) Willing and able to undergo phlebotomy for }\end{array}$ \\
$\begin{array}{l}\text { (vi) History of severe allergic reaction or anaphylaxis }(N=0) \\
\text { estimated GFR determination within } 30 \text { days of biopsy }\end{array}$ & $\begin{array}{l}\text { (vii) History of liver diseases other than HCV including iron overload }(N=0) \\
\text { (ix) Pregnant or nursing mother }(N=0)\end{array}$ \\
\hline
\end{tabular}

${ }^{1}$ Biopsies were performed for clinical care. ${ }^{2}$ Due to intraorbital shrapnel. GFR: glomerular filtration rate. Parenthesis () contains the number of potential subjects excluded for the criterion.

the pathology department per routine protocol, including Masson-trichrome staining. Clinical biopsy reports were generated by staff pathologists. Each clinical report included assessment of fibrosis severity (none, mild, moderate, severe, and cirrhosis); the clinically reported fibrosis severity was used for the block recruitment but not analyzed.

2.3. Qualitative and Quantitative Scoring of Histology. The trichrome-stained slides were further evaluated for research purposes. The entire slides were digitized using an APERIO ScanScope scanner (Aperio Technologies, Inc., Vista, CA). The digitized images were viewed using the Aperio ImageScope software and the fibrosis severity was scored qualitatively by histomorphology and quantitatively by digital image analysis.

Qualitative scoring was performed independently by three pathologists with expertise in liver pathology (MRP, $\mathrm{HM}$, and ZG). Without knowledge of clinical, MR imaging, or quantitative histology findings, each reader reviewed the digitized histology images, subjectively assessed the adequacy of each specimen, and assigned to each specimen a Metavir fibrosis score, F0-F4. The readers were blinded to each other's scores. Other histology features (e.g., necro-inflammation, steatosis, iron) were not recorded. To assess adequacy of specimen, one pathologist (MRP) counted the number of portal triads within each noncirrhotic specimen; portal triads were not counted in cirrhotic specimens due to architectural distortion. The total length of each specimen was recorded.

Quantitative scoring was performed by a hepatology research scientist (KI) using ImageScope software analysis tools, without knowledge of the clinical, MR imaging, or qualitative histology findings. Staining variability was corrected by digitally adjusting color saturation. Total specimen area was manually segmented, and the blue-stained pixels (representing collagen) were segmented using manual intensity thresholding. Percent (\%) collagen was calculated as the ratio of blue-stained to total specimen pixels.

2.4. MR Imaging. Subjects received SPIO (ferumoxides, Feridex, Bayer HealthCare Pharmaceuticals, Wayne, NJ) continuous intravenous infusion $(0.5 \mathrm{~mL} / \mathrm{kg})$ diluted in $100 \mathrm{~mL}$ of $5 \%$ dextrose solution, passed through a $5-\mu \mathrm{m}$ filter at 2$4 \mathrm{~mL} / \mathrm{min}$ over 30 minutes per manufacturer's instructions.
Thirty minutes after completion of SPIO infusion, subjects were scanned supine in a superconducting MR whole body system at 3T (GE Signa EXCITE HD, GE Medical Systems, Milwaukee, WI), with an 8-channel torso phase-array coil and a dielectric pad centered over the liver. Gadolinium-DTPA (gadopentetate dimeglumine, Magnevist, Bayer HealthCare Pharmaceuticals, Wayne, NJ) was injected intravenously $(0.1 \mathrm{mmol} / \mathrm{kg})$. Using a $2 \mathrm{D}$ chemically fat-saturated fast spoiled gradient-recalled echo (FSPGR) sequence without parallel imaging, four sets of axial CCE images of the liver were acquired during separate 18-28 second breath-holds, 4-10 minutes after Gd injection. In this 6-minute window, enhancement of the liver by the two agents (SPIO and Gd) is subjectively constant according to our clinical experience of CCE MR imaging in cirrhotic and noncirrhotic livers; moreover, the T1- and T2* shortening effects of gadopentetate and ferumoxides in liver may be assumed stable over this period from the known liver clearance rates of these agents [31-33]. The four image sets were acquired to help ensure that at least one set was free of visible motion artifacts. Imaging parameters included TR $100 \mathrm{~ms}$, TE $6 \mathrm{~ms}$, FA $70^{\circ}$, slice thickness $4 \mathrm{~mm}$, interslice gap $4 \mathrm{~mm}$, number of slices 5, and bandwidth $130 \mathrm{~Hz} /$ pixel. Two of the four image sets were acquired with $384 \times 224$ and two with $384 \times 256$ matrix. Field-of-view was adjusted to accommodate body habitus and breath-hold capacity. These parameters were selected to provide simultaneous T1- and T2* -weighting to exploit Gd- and SPIO-enhancement, respectively; adequate signal-to-noise ratio; high spatial resolution; and relatively short acquisition time. The Food and Drug Administration (IND number 75,579) approved off-label use of MagnevistFeridex combined contrast for this research study.

2.5. Image Processing and Texture Analysis. A radiology resident (TY) and a trained research assistant (CC) analyzed the CCE images without knowledge of clinical or biopsy findings. From the four CCE image sets, the set with the highest resolution and subjectively least motion artifact was selected. Representative CCE images of the liver (1-5 sections per subject) were exported in DICOM format. Using MATLAB (Mathworks, Natick, MA), a total of five nonoverlapping rectangular regions-of-interest (ROIs) of size $>100 \mathrm{~mm}^{2}$ were placed per subject within areas of subjectively uniform texture 
in the right hepatic lobe (Couinaud segments IV-VIII), avoiding artifacts, bile ducts, and vessels. Each ROI image was standardized by rotating to the Cartesian coordinate system with zero tilt-angle, interpolating to $0.5 \mathrm{~mm} /$ pixel resolution, removing bilinear spatial trend of signal intensities, and scaling to $0-1$ intensity range.

Gradient and Laplacian transformations (1st and 2nd spatial derivatives) were applied to each standardized ROI to generate additional "edge-enhanced" and "zero-crossing" texture patterns. For each untransformed (original) and transformed (gradient, Laplacian) ROI, 55 texture features were calculated as detailed in the supplementary materials available online at http://dx.doi.org/10.1155/2014/387653. These texture features represented five texture feature classes: pixel intensity histogram, Gaussian mixture model, autocorrelation, cooccurrence matrices, and Voronoi polygons. These classes were selected based on the expected imaging characteristics of fibrosis texture, as explained in the supplementary materials. For each subject, the texture features were averaged across the five ROI's to generate a set of 165 average texture features.

\subsection{Statistical Analyses}

2.6.1. Comparison of Histologic Scores. For each subject, the average, standard deviation (STD), and range of the Metavir scores of the three pathology readers were calculated. The interreader agreement was assessed by intraclass correlation coefficient (ICC, two-way analysis for precise agreement) and their 95\% confidence intervals (CIs) were calculated. ICC was also calculated for each pair of readers. The average Metavir scores of the three readers were compared to \%-collagen scores using Pearson correlation analysis.

2.6.2. Comparison of Texture and Histology. A biostatistician (TW) performed statistical analysis using the 165 texture features to predict qualitative (Metavir) and quantitative (\%collagen) fibrosis scores. A path-following algorithm for $L_{1}$ regularized linear model called GLM-path [34] with a Gaussian link (i.e., linear regression) was used to identify the optimal linear model of texture features that minimized the fibrosis prediction error for each number of predictors (i.e., features). The optimal number of predictors was determined by Akaike Information Criterion (AIC) [35]. Using the qualitative and quantitative fibrosis scores as the reference, two texture-based fibrosis prediction models were constructed, respectively. For each subject, the predicted qualitative (Metavir) and quantitative (\%-collagen) fibrosis scores were calculated using respective prediction models.

Pearson's correlation was used to evaluate the strength of the relationship between the predicted and histologic scores. Additionally, the performance of each prediction model for dichotomized classification was assessed using receiveroperating-characteristics (ROC) analysis using the average histologically determined Metavir score as the reference standard. At each of four classification thresholds (Metavir F1, F2, F3, and F4 for qualitative scoring; 5, 10, 15, and 20\% collagen for quantitative scoring), the classification accuracy, sensitivity, and specificity (and their CIs) were calculated at the predicted fibrosis score cutoff value that maximized the sum of sensitivity and specificity.

The regularization employed by the GLM-path algorithm is designed to minimize prediction error over independent validation datasets [34]. Therefore no dedicated validation procedure was performed in this proof-of-concept study. However, the algorithm may not necessarily minimize the prediction error of the test dataset itself; thus, some degree of mismatch between the predicted and actual fibrosis scores is expected.

\section{Results}

3.1. Subjects. Between August 2007 and March 2009, 52 newly diagnosed HCV-positive adults (age 51.2 \pm 6.3 years, 38 male, 12 female) with recent or planned liver biopsy were recruited for CCE imaging. Six subjects were excluded (Table 1). The remaining 46 subjects formed the study group. All subjects completed the MR examination without serious adverse effects. At least one CCE image set was subjectively adequate in quality for further image analyses in each subject.

3.2. Qualitative versus Quantitative Histology. Examples of biopsy specimens are shown in Figure 3. The histology specimen's average \pm STD [range] of the total length and the number of portal triads were $21.9 \pm 9.8 \mathrm{~mm}$ [6.7-44.2] and $14.2 \pm 6.0$ [4-28], respectively.

Figure 4(a) shows the histogram of qualitative Metavir scores assigned by the three readers. The 3-reader agreement was good with ICC of 0.772 (95\% CI [0.653-0.859]). Pairwise ICCs were $0.727,0.768$, and 0.831 , depending on the reader pairs. All readers agreed that all biopsy specimens were adequate.

Figure 4(b) shows the histogram of quantitative \%collagen rounded to the nearest $5 \%$. Over half the subjects had rounded $\%$-collagen $\leq 5 \%$. As shown in Figure 5 the relationship between the qualitative (average Metavir) and quantitative (\%-collagen) scores was curvilinear, as has been observed by others $[36,37]$. Log-linear plot of quantitative ( $y$-axis) and qualitative ( $x$-axis) scores demonstrated significant linear correlation with Pearson's $r=0.81(P<0.001)$.

3.3. Image Texture versus Histology. The liver image textures of representative subjects are shown in Figure 6 with their respective qualitative (Metavir) and quantitative (\%-collagen) scores.

Using qualitative histology as the reference, GLM-path analysis identified a set of 6 texture features predictive of Metavir fibrosis scores (Table 2). As shown in Figure 7(a), the Metavir score predicted by a 6-feature model linearly correlated with the average Metavir scores of the three readers with $r=0.698(P<0.001)$. Table 3 summarizes the ROC analysis results at each classification threshold. AUCs were 0.8140.976 , sensitivities $0.659-1.000$, specificities $0.778-0.930$, and accuracies $0.674-0.935$, depending on the classification threshold. 

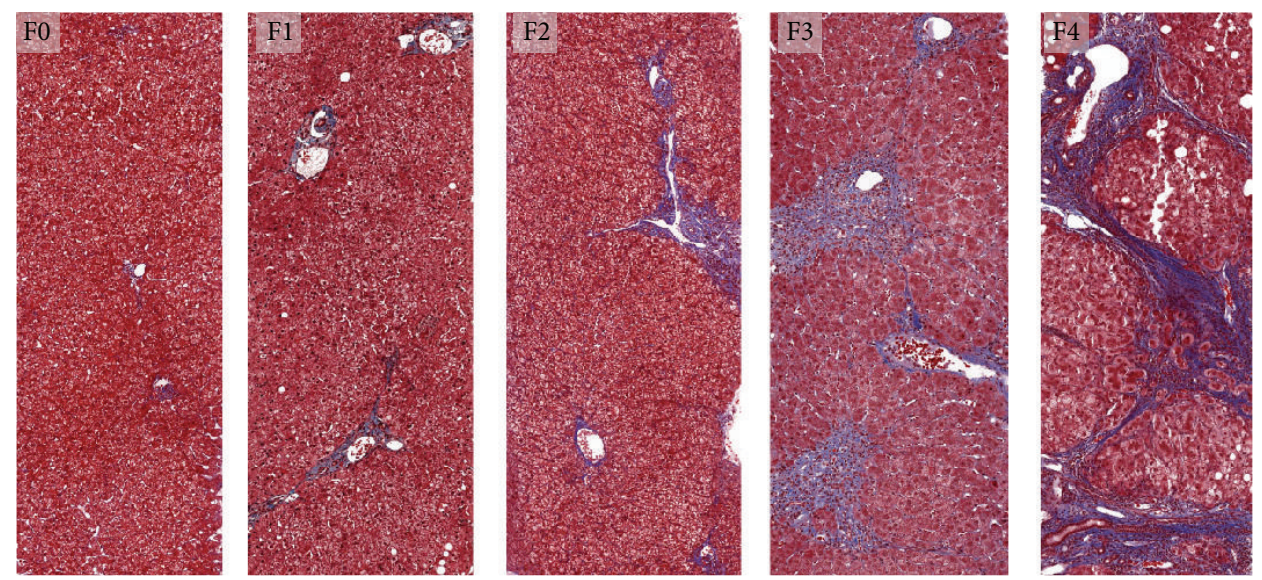

Figure 3: Histologic assessment of liver HCV-related fibrosis. Liver biopsy specimen from subjects with chronic HCV infection, stained with Masson-trichrome. F0 (absent fibrosis), F1 (stellate enlargement of portal tracts), F2 (enlarged portal tracts with rare septa), F3 (numerous septa without cirrhosis), and F4 (cirrhosis) according to Metavir scoring system. Trichrome stains fibrosis blue.

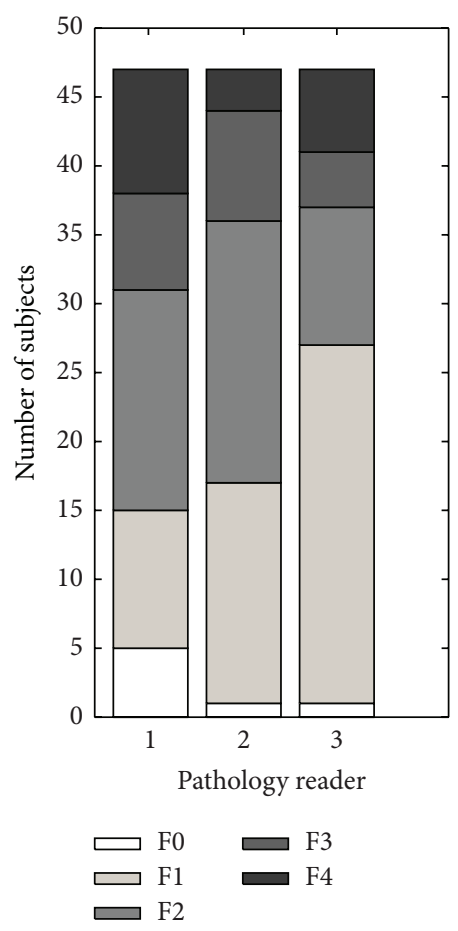

(a)

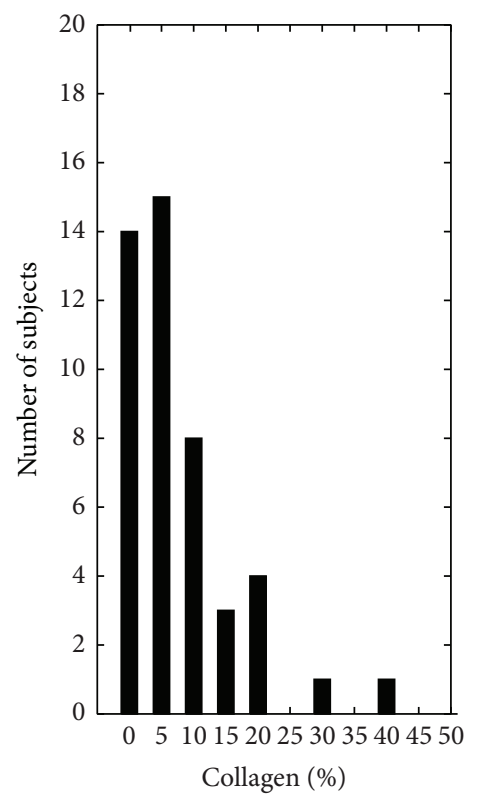

(b)

Figure 4: Fibrosis severity distribution. The study group's histograms by Metavir fibrosis score (left) and \%-collagen (right). The most common Metavir fibrosis score was F1 or F2 depending on the reader. Nine subjects (19\%; 9/46) had a score of F4 (cirrhosis) from at least one reader. $\%$-collagen is rounded to the nearest $5 \%$.

Using quantitative histology as the reference, GLM-path analysis identified another set of 6 texture features predictive of \%-collagen scores (Table 4). As shown in Figure 5 (LEFT) the \%-collagen score predicted by the 6 -feature model linearly correlated with \%-collagen score of histology with $r=0.757(P<0.001)$. Table 5 summarizes the ROC analysis results at threshold values at 5,20,15, and $20 \%$ fibrosis. AUCs were $0.742-0.950$, sensitivities $0.688-1.000$, specificities $0.679-0.857$, and accuracies $0.696-0.848$, depending on the classification threshold.
Identified texture features were similar but not identical between qualitative and quantitative prediction models (Tables 2 and 4). Two classes of texture features were common to both Gaussian-mixture model and Voronoi polygons. One class of texture features (pixel intensity histogram) was predictive only for qualitative scores. Texture features of both untransformed and transformed ROI images were found to be predictive. For illustration purposes, these texture classes derived from a ROI in a cirrhotic subject are shown in Figure 8. 


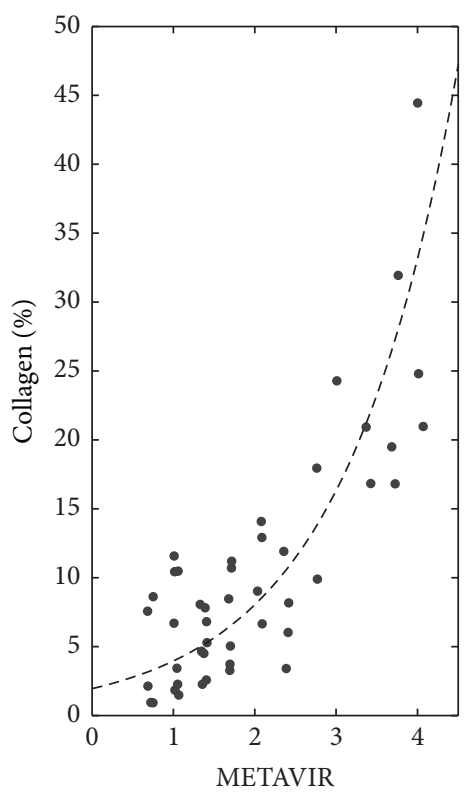

(a)

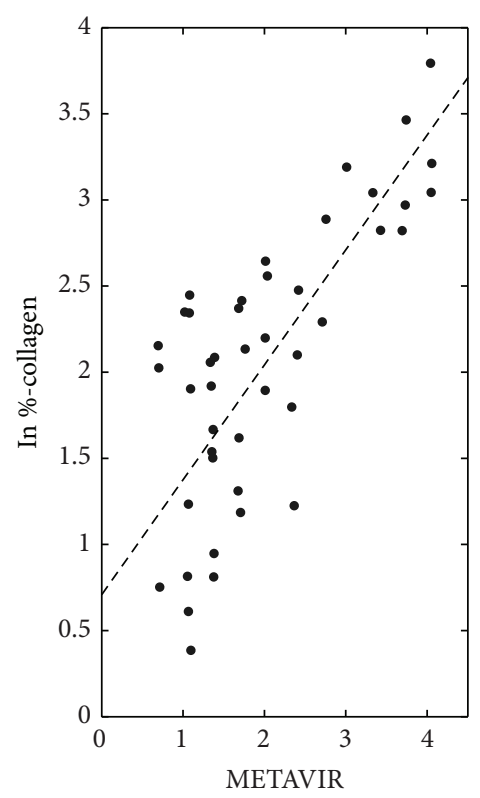

(b)

FIGURE 5: Comparison between quantitative versus qualitative histology. Average Metavir of 3 pathology readers versus (a) raw \%-collagen (b) and natural logarithm of \%-collagen. As shown in (a), the relationship between quantitative and qualitative histology scores is curvilinear. Pearson's correlation coefficient $(r)$ of plot B is 0.81 , with $P<0.001$.

TABLE 2: Fibrosis prediction model parameters (texture versus Metavir).

\begin{tabular}{llll}
\hline & Source image & Texture class & Texture feature \\
\hline 1 & Original & Pixel intensity histogram & Mean pixel intensity \\
2 & Original & Gaussian mixture model & STD of the lower intensity pixels \\
3 & Original & Gaussian mixture model & AIC of two-Gaussian fit/AIC of single-Gaussian fit \\
4 & Original & Voronoi polygons & STD of the 1st order inertial moment \\
5 & Gradient & Voronoi polygons & Mean of the 2nd order inertial moment \\
6 & Laplacian & Pixel intensity histogram & Mode/interquartile range \\
\hline
\end{tabular}

Six most predictive texture features, from strongest to weakest. Keys: STD: standard deviation, AIC: Akaike Information Criterion, inertial moments: mathematical description the shape/area of the Voronoi polygons (see supplementary materials).

TABLE 3: Receiver operating characteristics (texture versus Metavir).

\begin{tabular}{lccccr}
\hline Classification & Cutoff & Area under curve & Sensitivity & Specificity & Accuracy \\
\hline $\mathrm{F}<1$ versus $\mathrm{F} \geq 1$ & 1.805 & $0.814[0.6540 .975]$ & $0.659[0.5130 .804]$ & $0.800[0.4491 .000]$ & $0.674[0.5240 .797]$ \\
$\mathrm{F}<2$ versus $\mathrm{F} \geq 2$ & 1.916 & $0.889[0.7830 .994]$ & $0.895[0.7571 .000]$ & $0.778[0.6210 .919]$ & $0.826[0.6860 .916]$ \\
$\mathrm{F}<3$ versus $\mathrm{F} \geq 3$ & 2.060 & $0.862[0.7011 .000]$ & $0.778[0.5061 .000]$ & $0.784[0.6510 .916]$ & $0.783[0.6150 .867]$ \\
$\mathrm{F}<4$ versus $\mathrm{F}=4$ & 2.174 & $0.976[0.8551 .000]$ & $1.000[0.9071 .000]$ & $0.930[0.8541 .000]$ & $0.935[0.7880 .974]$ \\
\hline
\end{tabular}

Cutoff: the operating point on the ROC curve closest to $(0,1)$, the point of maximum sensitivity and specificity. [ ] $-95 \%$ confidence interval. The mismatch between the texture-based cutoff and the histologic classification threshold is expected (see text).

\section{Discussion}

This study prospectively assessed liver fibrosis in HCVinfected adults noninvasively using quantitative texture analysis of CCE MR images. Liver biopsy was used as the reference standard. Fibrosis severity was scored qualitatively (Metavir) and quantitatively (\%-collagen). The study design closely simulated a typical clinical situation, in which a newly diagnosed HCV-positive patient without clinically overt cirrhosis requires assessment of liver fibrosis.
We utilized a CCE MR imaging technique, in which SPIOs and an extracellular Gd-based agent are administered sequentially. Prior studies suggested complimentary effects of SPIO and Gd for visualizing fibrosis. SPIOs accumulate by phagocytosis in Kupffer cells in the hepatic lobules, causing T2* ${ }^{*}$-related negative enhancement. Extracellular Gd-based agents such as Gd-DTPA distribute to the interstitial space of the fibrotic perilobular septa, causing T1-related positive enhancement. The result is a linear meshwork of high-signal perilobular septa against a background of low-signal lobules, 

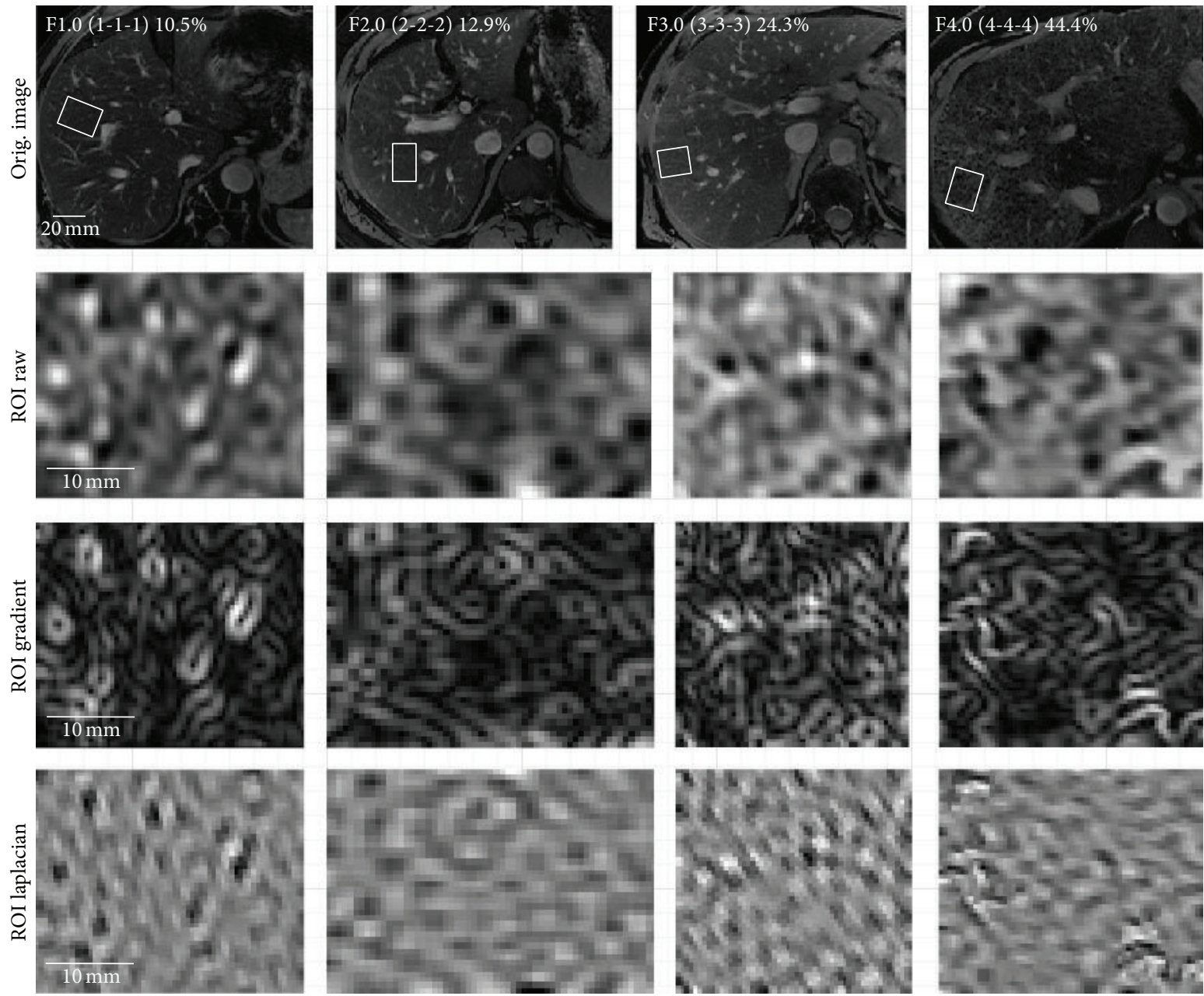

FIGURE 6: Regions-of-interest on CCE MR images in four subjects. Regions-of-interest drawn on CCE MR images in four representative subjects. Average Metavir (and individual reader METAVIR) and \%-collagen scores are shown. Subjectively, high-signal reticular texture becomes increasingly conspicuous and disorganized with increasing Metavir and \%-collagen scores. CCE: combined contrast enhanced.

TABLE 4: Fibrosis prediction model parameters (texture versus \%-collagen).

\begin{tabular}{llll}
\hline & Source image & Texture class & Texture feature \\
\hline 1 & Original & Gaussian mixture model & STD of the lower intensity pixels \\
2 & Original & Voronoi polygons & Mean of the 2nd order inertial moment \\
3 & Original & Voronoi polygons & STD of the 1st order inertial moment \\
5 & Gradient & Voronoi polygons & Mean of the 2nd order inertial moment \\
6 & Gradient & Gaussian mixture model & STD of the lower intensity pixels \\
\hline
\end{tabular}

Six most predictive texture features, from strongest to weakest. Keys: STD: standard deviation, AIC: Akaike Information Criterion, inertial moments: mathematical description the shape/area of the Voronoi polygons (see supplementary materials).

TABLE 5: Receiver operating characteristics (texture versus \%-collagen).

\begin{tabular}{lccccc}
\hline Classification & Cutoff & Area under curve & Sensitivity & Specificity & Accuracy \\
\hline$<5 \%$ versus $\geq 5 \%$ & 9.315 & $0.806[0.6800 .932]$ & $0.688[0.5270 .848]$ & $0.857[0.6741 .000]$ & $0.739[0.5920 .850]$ \\
$<10 \%$ versus $\geq 10 \%$ & 9.807 & $0.742[0.5890 .895]$ & $0.722[0.5150 .929]$ & $0.679[0.5060 .852]$ & $0.696[0.5470 .815]$ \\
$<15 \%$ versus $\geq 15 \%$ & 10.500 & $0.894[0.7581 .000]$ & $0.900[0.7141 .000]$ & $0.750[0.6090 .891]$ & $0.783[0.6380 .884]$ \\
$<20 \%$ versus $\geq 20 \%$ & 11.234 & $0.950[0.8261 .000]$ & $1.000[0.9071 .000]$ & $0.825[0.7070 .943]$ & $0.848[0.6860 .916]$ \\
\hline
\end{tabular}

Cutoff: the operating point on the ROC curve closest to $(0,1)$, the point of maximum sensitivity and specificity. [ ]-95\% confidence interval. The mismatch between the texture-based cutoff and the histologic classification threshold is expected (see text). 


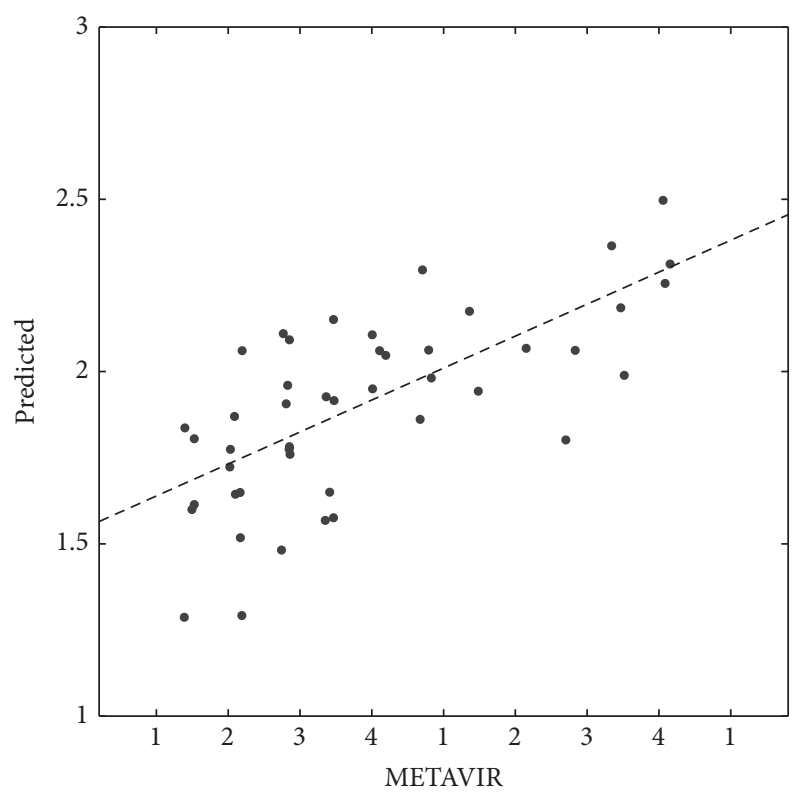

(a)

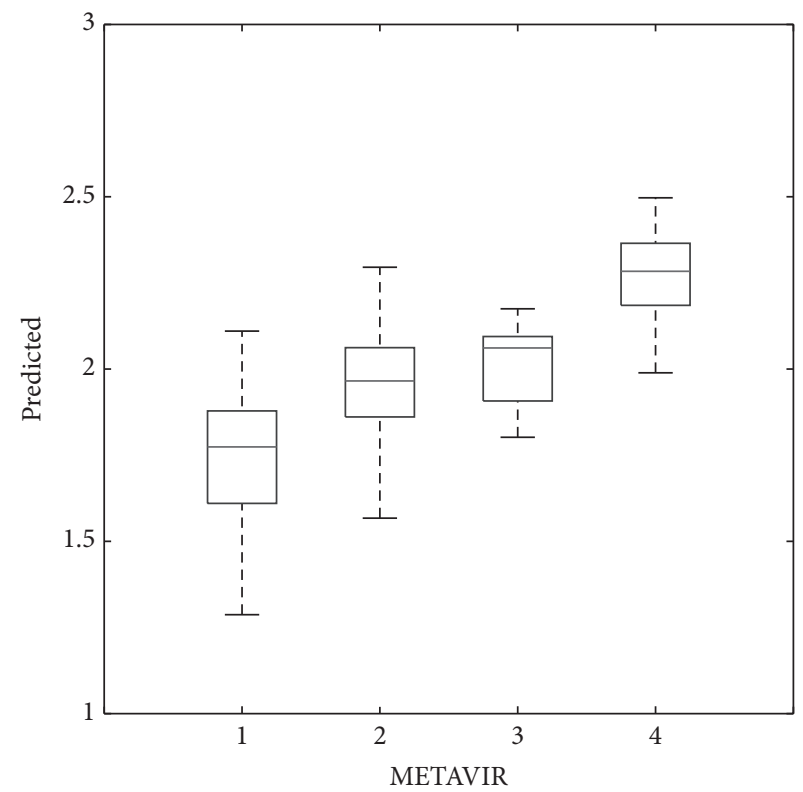

(b)

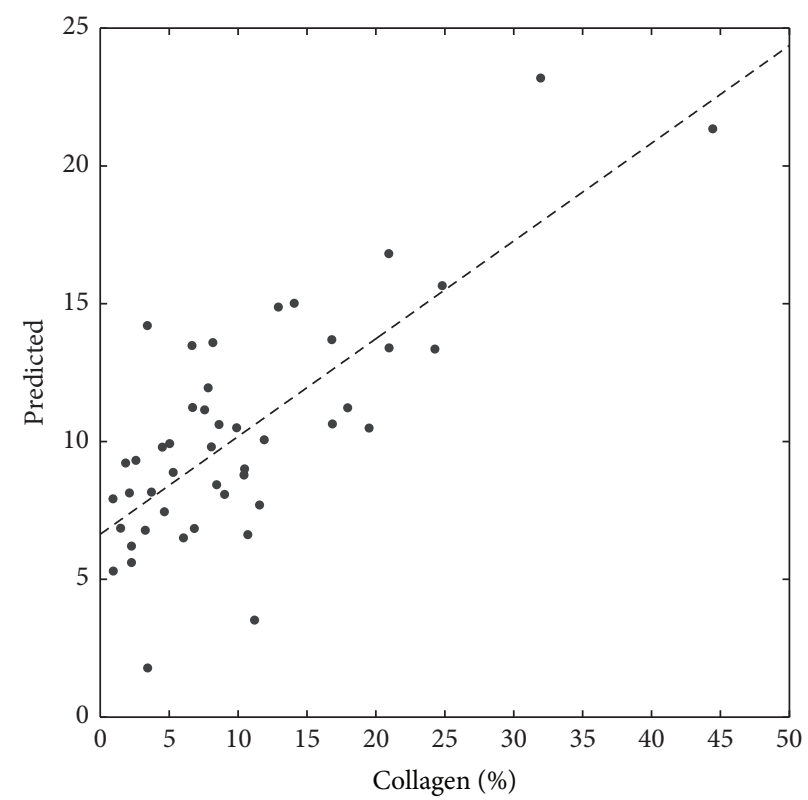

(c)

Figure 7: Texture versus histologic fibrosis scores. (a) The correlation between the texture and qualitative histology score (average Metavir) is statistically significant with $0.698(P<0.001)$ with best-fit line of slope 1.546 and intercept 0.186 . (b) Box-plot of texture score versus rounded average Metavir. Spearman's correlation is significant at $\rho=0.635(P<0.001)$. (c) The correlation between texture and quantitative histology score (percent-fibrosis) is statistically significant with $0.767(P<0.001)$, with best-fit line of slope 0.355 and intercept 6.636 . The nonunit slope and nonzero intercept are attributable in part to the regularization procedure employed by the GLM-path algorithm (see text).

producing a reticular texture pattern that subjectively becomes more conspicuous with increasing fibrosis severity $[26,27]$.

We found that CCE MR image texture of the liver can be objectively quantified to predict fibrosis severity. The abnormal texture was detectable at early fibrosis stage, for example, F $>2$ Metavir score or $>15 \%$-collagen with accuracy of 0.826 and 0.783 , respectively. The predicted fibrosis scores correlated with but did not exactly match the corresponding histologically determined scores. The imperfect agreement between predicted and actual fibrosis scores is likely due to three factors: intrinsic inaccuracy of the texture-based technique used in our study, expected mismatch due to the regularization procedure employed by GLM-path (explained earlier), and intrinsic inaccuracy of liver biopsy as a reference standard (explained later). 

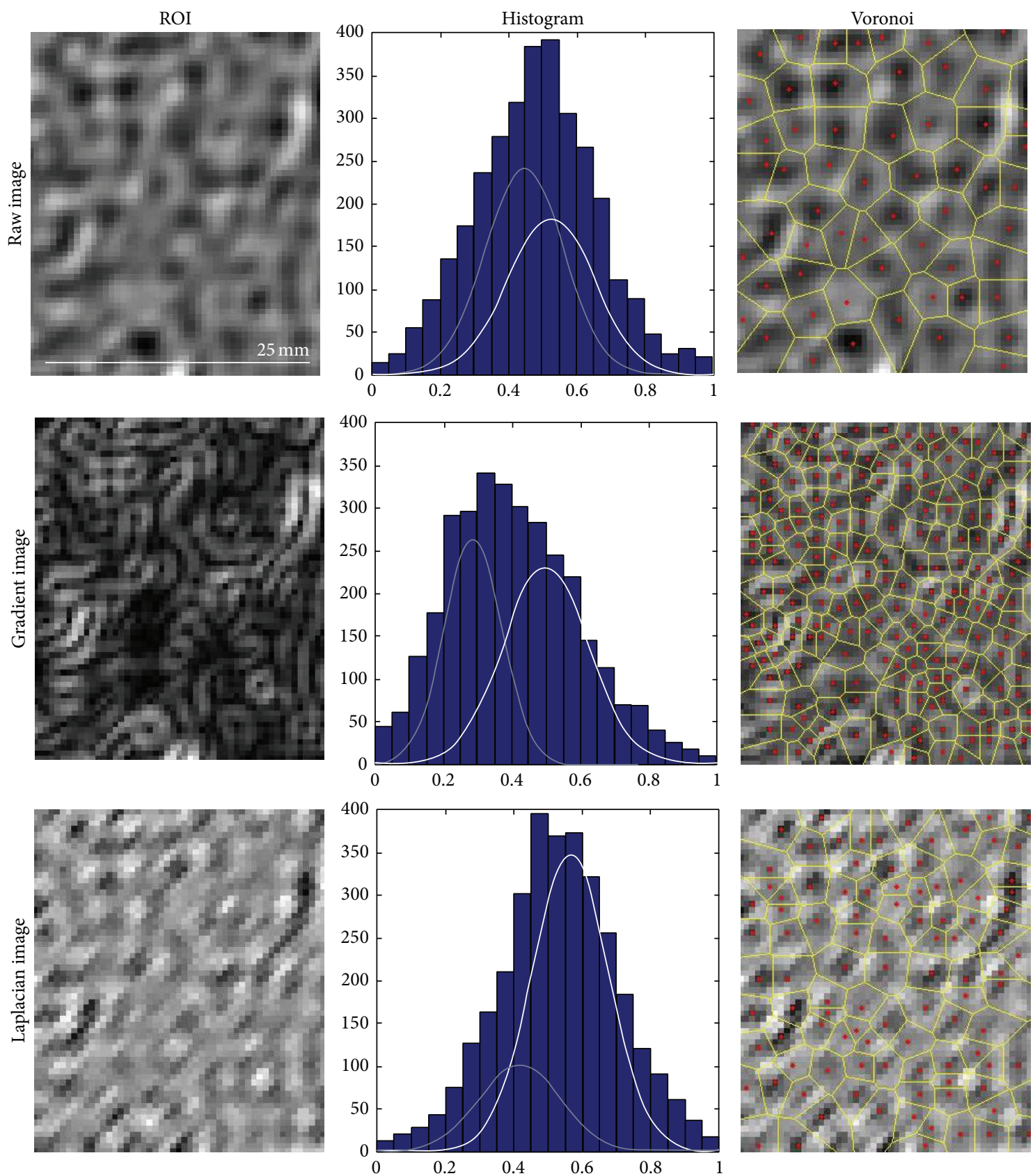

(a)

(b)

(c)

FiguRE 8: Illustrative examples of texture feature classes for fibrosis prediction. A 54 year-old female with cirrhosis. (c) Standardized regions of interest (ROI) images, without transformation (raw), with gradient and Laplacian transformations. (b) Corresponding pixel intensity histogram and its Gaussian mixture model fit with two normal populations. (a) Voronoi polygons constructed on the corresponding ROI images. These texture classes were found to be predictive of liver fibrosis on CCE images (see text). For each texture class (intensity histogram, Gaussian mixture, and Voronoi polygons), relevant statistics were calculated as detailed in supplementary materials and were used for fibrosis prediction.

Gaussian mixture models and Voronoi polygons were found to be predictive texture classes by both qualitative and quantitative histology prediction models. This is consistent with the postulated complimentary effects of SPIO and Gd producing the reticular enhancement pattern in fibrotic livers. A Gaussian mixture model fits two normal distributions, each with its own mean and variance, to the overall pixel intensity histogram. On a CCE image, the liver contains two populations of pixels, one comprised of low-signal SPIOcontaining pixels devoid of fibrosis and the other of highsignal Gd-containing pixels in fibrotic septa. With progression of fibrosis, the proportion of high-signal Gd-containing 
pixels (i.e., fibrosis) increases and, therefore, the pixel intensity histogram is better fitted by a mixture of two Gaussian distributions than a single Gaussian. The Voronoi polygon algorithm generates a tessellation of polygons that "carves" the liver parenchyma into low-intensity nodules, thereby objectively modeling the reticular texture seen subjectively in progressive fibrosis.

Another MR-based technique, MR elastography (MRE), is increasing in popularity and availability for noninvasive assessment of liver fibrosis. This technique measures the biomechanical stiffness of the liver, which increases as a consequence of fibrosis [38]. In a retrospective study in HCVinfected population [39], the reported AUC, sensitivity, and specificity of MRE in detecting clinically significant fibrosis $(\mathrm{F} \geq 2$ ) was $0.986,0.885$, and 1.00 , respectively, similar to slightly higher than those of the CCE texture method. However, texture-based methods may have a theoretical advantage of more direct visualization of fibrosis while MRE measures the tissue biomechanical sequela of fibrosis. Another practical advantage of texture-based methods is that they can be implemented on any clinical scanner using standard sequences, while MRE requires dedicated hardware (mechanical wave transducer) and sequences. Disadvantages of texture-based methods are the need for intravenous access and injection of contrast agents, including two agents for the CCE technique described here. Also, visualization of subtle reticulations associated with early fibrosis is sensitive to patient motion; consequently we obtained four CCE image sets in separate breath-holds, to ensure that at least one set was motionfree. As motion correction/minimization techniques become more robust and clinically available, it may be possible to acquire images during free breathing with higher signal-tonoise ratio and spatial resolution.

A limitation of this study is the use of single liver biopsy as the reference standard. A typical core biopsy $\left(\sim 30 \mathrm{~mm}^{3}\right)$ samples only $1 / 50,000$ of the liver and is significantly smaller than the imaging ROI $\left(>400 \mathrm{~mm}^{3}\right)$ used in the texture analysis. Also, biopsied sites are difficult to colocalize with imaging ROIs, which is relevant because the severity of fibrosis can be heterogeneous across the liver. An error frequency of up to $33 \%$ has been reported for differences in $\geq$ one fibrosis stage and cirrhosis may be missed in $10-30 \%$ of blind biopsies [40]. Thus even a "perfect" fibrosis prediction method may have only moderate observed accuracy in binary classification if single-biopsy histology is used as the reference. Obtaining multiple biopsies may reduce errors in the reference standard, but increases the complication risk and was not feasible in this study. Considering these limitations, moderate accuracy of CCE MR imaging is appropriate and expected. Determination of the true accuracy of fibrosis imaging may require histologic evaluation of larger specimens than those obtained by percutaneous biopsy. Another consideration for the accuracy of biopsy as the reference standard is observer bias [10]. To minimize the observer bias and increase the accuracy of fibrosis staging, this study used the average Metavir score of three hepatopathologists' independent interpretations as the reference standard. While averaging of an ordinal fibrosis score is less than ideal, it is arguably the most valid fibrosis severity metric available from a single biopsy specimen. Such a practice is not uncommon in hepatology literature [41-44]. As average Metavir score is expected to preserve the rank-order relationship of the fibrosis severity, it should be sufficient to mathematically construct a valid fibrosis prediction model.

While this study suggested a promising indication for SPIO agents in liver fibrosis imaging, ferumoxides were withdrawn from the US market in 2009. Recently, another intravenously injectable SPIO-based drug, ferumoxytol (Feraheme, AMAG Pharmaceuticals, Lexington, MA) has been FDA-approved for iron-deficiency therapy and early data on its application as contrast agent for MR imaging are promising [45]. While this new drug likely has similar negativecontrast effects in the liver as ferumoxides, further studies will be necessary to evaluate its effectiveness in CCE imaging.

In summary, this proof-of-concept prospective study showed that CCE MR imaging and quantitative texture analysis may permit noninvasive assessment of liver fibrosis in $\mathrm{HCV}$-infected adults. MR image texture is a potential noninvasive biomarker of liver fibrosis and, with further technical refinement and validation, may provide a new tool in clinical management and research in $\mathrm{HCV}$-infected patients.

\section{Conflict of Interests}

The contents of this work are solely the responsibility of the authors and do not necessarily represent the official views of the funding agencies.

\section{Acknowledgments}

This work was funded in part by grants from the Radiological Society of North America R\&E Foundation Research Resident Award no. RR0726, the National Institutes of Health NCMHD EXPORT P60 MD00220, and Bayer Healthcare Grant no. 070525.

\section{References}

[1] K. D. Kochanek, S. L. Murphy, R. N. Anderson, and C. Scott, "Deaths: final data for 2002," National Vital Statistics Reports, vol. 53, no. 5, pp. 1-115, 2004.

[2] Y.-S. Lim and W. R. Kim, "The global impact of hepatic fibrosis and end-stage liver disease," Clinics in Liver Disease, vol. 12, no. 4, pp. 733-746, 2008.

[3] S. Vong and B. P. Bell, "Chronic liver disease mortality in the United States, 1990-1998," Hepatology, vol. 39, no. 2, pp. 476483, 2004.

[4] G. L. Armstrong, A. Wasley, E. P. Simard, G. M. McQuillan, W. L. Kuhnert, and M. J. Alter, "The prevalence of hepatitis C virus infection in the United States, 1999 through 2002," Annals of Internal Medicine, vol. 144, no. 10, pp. 705-714, 2006.

[5] I. M. Arias, The Liver: Biology and Pathobiology, John Wiley \& Sons, Chichester, UK, 5th edition, 2009.

[6] L. Peters and J. K. Rockstroh, "Biomarkers of fibrosis and impaired liver function in chronic hepatitis C: how well do they predict clinical outcomes?" Current Opinion in HIV and AIDS, vol. 5, no. 6, pp. 517-523, 2010. 
[7] G. Germani, P. Hytiroglou, A. Fotiadu, A. K. Burroughs, and A. P. Dhillon, "Assessment of fibrosis and cirrhosis in liver biopsies: an update," Seminars in Liver Disease, vol. 31, no. 1, pp. 82-90, 2011.

[8] P. Marcellin, "Hepatitis B and hepatitis C in 2009," Liver International, vol. 29, supplement 1, pp. 1-8, 2009.

[9] P. Bedossa and T. Poynard, "An algorithm for the grading of activity in chronic hepatitis C," Hepatology, vol. 24, no. 2, pp. 289-293, 1996.

[10] "Intraobserver and interobserver variations in liver biopsy interpretation in patients with chronic hepatitis C. The French METAVIR Cooperative Study Group," Hepatology, vol. 20, no. 1, part 1, pp. 15-20, 1994.

[11] J. Perrault, D. B. McGill, B. J. Ott, and W. F. Taylor, "Liver biopsy: complications in 1000 inpatients and outpatients," Gastroenterology, vol. 74, no. 1, pp. 103-106, 1978.

[12] P. Thampanitchawong and T. Piratvisuth, "Liver biopsy: complications and risk factors," World Journal of Gastroenterology, vol. 5, no. 4, pp. 301-304, 1999.

[13] P. Halfon, F. Imbert-Bismut, D. Messous et al., "A prospective assessment of the inter-laboratory variability of biochemical markers of fibrosis (FibroTest) and activity (ActiTest) in patients with chronic liver disease," Comparative Hepatology, vol. 1, article 3, 2002.

[14] E. Rossi, L. Adams, A. Prins et al., "Validation of the FibroTest biochemical markers score in assessing liver fibrosis in hepatitis C patients," Clinical Chemistry, vol. 49, no. 3, pp. 450-454, 2003.

[15] H. Saito, S. Tada, N. Nakamoto et al., "Efficacy of non-invasive elastometry on staging of hepatic fibrosis," Hepatology Research, vol. 29, no. 2, pp. 97-103, 2004.

[16] A. Ziolkowski, M. Wylezol, M. Kukla et al., "The comparison of scoring scales for liver biopsy assessment in morbidly obese patients undergoing bariatric surgery," Obesity Surgery, vol. 15, no. 9, pp. 1309-1314, 2005.

[17] L. Huwart, F. Peeters, R. Sinkus et al., "Liver fibrosis: non-invasive assessment with MR elastography," NMR in Biomedicine, vol. 19, no. 2, pp. 173-179, 2006.

[18] O. Rouvière, M. Yin, M. A. Dresner et al., "MR elastography of the liver: preliminary results," Radiology, vol. 240, no. 2, pp. 440-448, 2006.

[19] M. Yin, J. A. Talwalkar, K. J. Glaser et al., "Assessment of hepatic fibrosis with magnetic resonance elastography," Clinical Gastroenterology and Hepatology, vol. 5, no. 10, pp. 1207.e21213.e2, 2007.

[20] C. Aubé, P. X. Racineux, J. Lebigot et al., "Diagnosis and quantification of hepatic fibrosis with diffusion weighted MR imaging: preliminary results," Journal de Radiologie, vol. 85, no. 3, pp. 301-306, 2004.

[21] M. Koinuma, I. Ohashi, K. Hanafusa, and H. Shibuya, "Apparent diffusion coefficient measurements with diffusion-weighted magnetic resonance imaging for evaluation of hepatic fibrosis," Journal of Magnetic Resonance Imaging, vol. 22, no. 1, pp. 80-85, 2005.

[22] J. A. Talwalkar, M. Yin, J. L. Fidler, S. O. Sanderson, P. S. Kamath, and R. L. Ehman, "Magnetic resonance imaging of hepatic fibrosis: emerging clinical applications," Hepatology, vol. 47, no. 1, pp. 332-342, 2008.

[23] G. Elizondo, R. Weissleder, D. D. Stark et al., "Hepatic cirrhosis and hepatitis: MR imaging enhanced with superparamagnetic iron oxide," Radiology, vol. 174, no. 3, pp. 797-801, 1990.
[24] T. Fujita, K. Ito, K. Honjo, H. Okazaki, T. Matsumoto, and N. Matsunaga, "Hepatic parenchymal enhancement in the cirrhotic liver: evaluation by triple-phase dynamic MRI," Abdominal Imaging, vol. 27, no. 1, pp. 29-33, 2002.

[25] K. M. Vitellas, M. T. Tzalonikou, W. F. Bennett, K. K. Vaswani, and J. G. Bova, "Cirrhosis: spectrum of findings on unenhanced and dynamic gadolinium-enhanced MR imaging," Abdominal Imaging, vol. 26, no. 6, pp. 601-615, 2001.

[26] D. A. Aguirre, C. A. Behling, E. Alpert, T. I. Hassanein, and C. B. Sirlin, "Liver fibrosis: noninvasive diagnosis with double contrast material-enhanced MR imaging," Radiology, vol. 239, no. 2, pp. 425-437, 2006.

[27] F. Hughes-Cassidy, A. D. Chavez, A. Schlang et al., "Superparamagnetic iron oxides and low molecular weight gadolinium chelates are synergistic for direct visualization of advanced liver fibrosis," Journal of Magnetic Resonance Imaging, vol. 26, no. 3, pp. 728-737, 2007.

[28] H. Kato, M. Kanematsu, X. Zhang et al., "Computer-aided diagnosis of hepatic fibrosis: preliminary evaluation of MRI texture analysis using the finite difference method and an artificial neural network," American Journal of Roentgenology, vol. 189, no. 1, pp. 117-122, 2007.

[29] G. Bahl, D. A. Aguirre, G. Motta, A. Fotinos, G. Rescinito, and C. Sirlin, "Quantitative texture analysis of liver fibrosis on double-contrast enhanced gradient recalled echo images," in Proceedings of the RSNA Annual Meeting, Chicago, Ill, USA, 2005.

[30] G. Bahl, I. Cruite, T. Wolfson et al., "Noninvasive classification of hepatic fibrosis based on texture parameters from double contrast-enhanced magnetic resonance images," Journal of Magnetic Resonance Imaging, vol. 36, no. 5, pp. 1154-1161, 2012.

[31] P. F. Hahn and S. Saini, "Liver-specific MR imaging contrast agents," Radiologic Clinics of North America, vol. 36, no. 2, pp. 287-297, 1998.

[32] Y. Gandon, J.-F. Heautot, F. Brunet, D. Guyader, Y. Deugnier, and M. Carsin, "Superparamagnetic iron oxide: clinical timeresponse study," European Journal of Radiology, vol. 12, no. 3, pp. 195-200, 1991.

[33] S. Aime and P. Caravan, "Biodistribution of gadolinium-based contrast agents, including gadolinium deposition," Journal of Magnetic Resonance Imaging, vol. 30, no. 6, pp. 1259-1267, 2009.

[34] M. Y. Park and T. Hastie, " $L_{1}$-regularization path algorithm for generalized linear models," Journal of the Royal Statistical Society B, vol. 69, no. 4, pp. 659-677, 2007.

[35] H. Akaike, "A new look at the statistical model identification," IEEE Transactions on Automatic Control, vol. 19, no. 6, pp. 716723, 1974.

[36] V. Calvaruso, A. K. Burroughs, R. Standish et al., "Computerassisted image analysis of liver collagen: relationship to Ishak scoring and hepatic venous pressure gradient," Hepatology, vol. 49, no. 4, pp. 1236-1244, 2009.

[37] M. J. O’Brien, N. M. Keating, S. Elderiny et al., “An assessment of digital image analysis to measure fibrosis in liver biopsy specimens of patients with chronic hepatitis C," The American Journal of Clinical Pathology, vol. 114, no. 5, pp. 712-718, 2000.

[38] S. K. Venkatesh and R. L. Ehman, "Magnetic resonance elastography of liver," Magnetic Resonance Imaging Clinics of North America, vol. 22, no. 3, pp. 433-446, 2014.

[39] S. Ichikawa, U. Motosugi, T. Ichikawa et al., "Magnetic resonance elastography for staging liver fibrosis in chronic hepatitis C," Magnetic Resonance in Medical Sciences, vol. 11, no. 4, pp. 291-297, 2012. 
[40] D. S. Manning and N. H. Afdhal, "Diagnosis and quantitation of fibrosis," Gastroenterology, vol. 134, no. 6, pp. 1670-1681, 2008.

[41] M. L. Shiffman, R. T. Stravitz, M. J. Contos et al., "Histologic recurrence of chronic hepatitis $\mathrm{C}$ virus in patients after living donor and deceased donor liver transplantation," Liver Transplantation, vol. 10, no. 10, pp. 1248-1255, 2004.

[42] J. C. Hoefs, M. L. Shiffman, Z. D. Goodman, D. E. Kleiner, J. L. Dienstag, and A. M. Stoddard, "Rate of progression of hepatic fibrosis in patients with chronic hepatitis C: results from the HALT-C trial," Gastroenterology, vol. 141, no. 3, pp. 900.e2908.e2, 2011.

[43] A. Monto, S. Kakar, L. M. Dove, A. Bostrom, E. L. Miller, and T. L. Wright, "Contributions to hepatic fibrosis in HIV-HCV coinfected and HCV monoinfected patients," The American Journal of Gastroenterology, vol. 101, no. 7, pp. 1509-1515, 2006.

[44] R. Malekzadeh, M. Mohamadnejad, and N. Rakhshani, "Reversibility of cirrhosis in chronic hepatitis B," Clinical Gastroenterology and Hepatology, vol. 2, no. 4, pp. 344-347, 2004.

[45] M. R. Bashir, L. Bhatti, D. Marin, and R. C. Nelson, "Emerging applications for ferumoxytol as a contrast agent in MRI," Journal of Magnetic Resonance Imaging, 2014. 


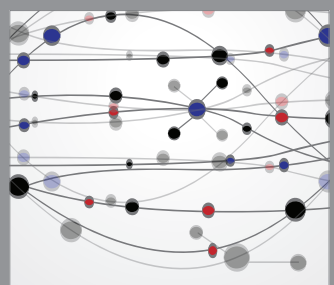

The Scientific World Journal
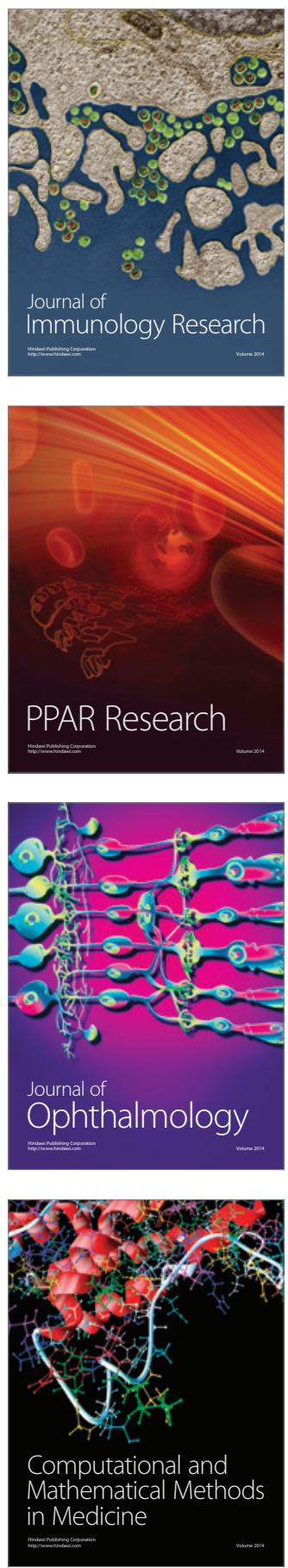

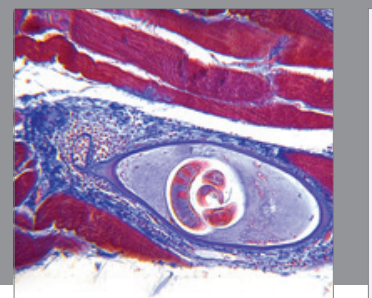

Gastroenterology

Research and Practice
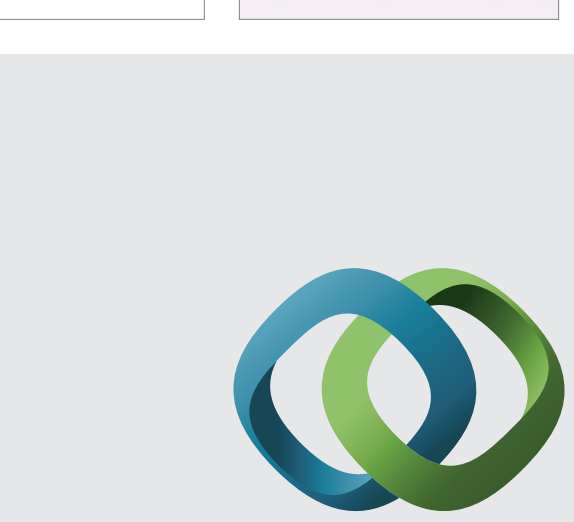

\section{Hindawi}

Submit your manuscripts at

http://www.hindawi.com
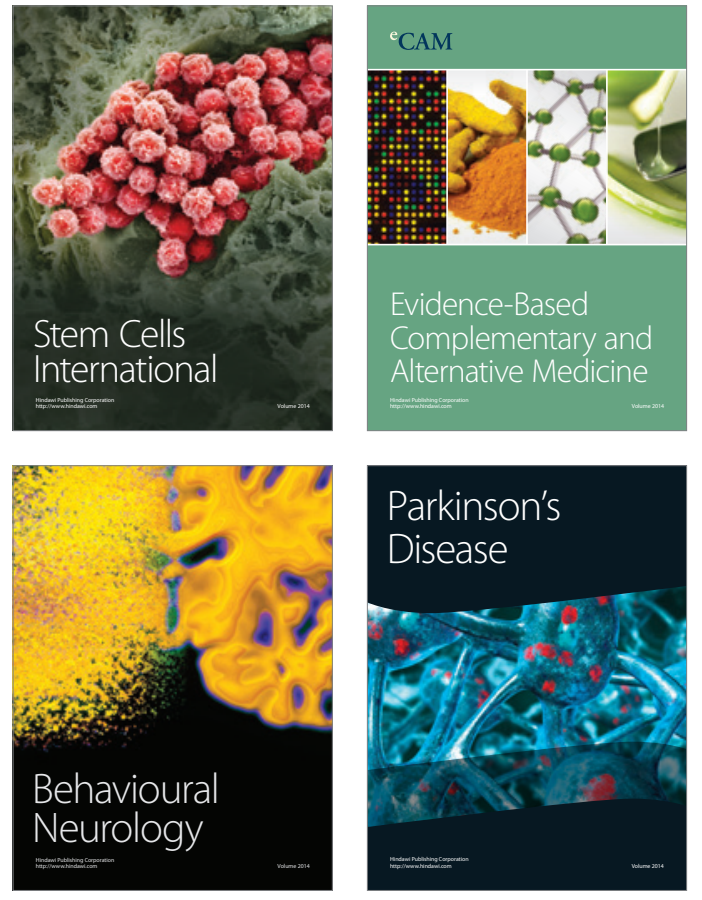
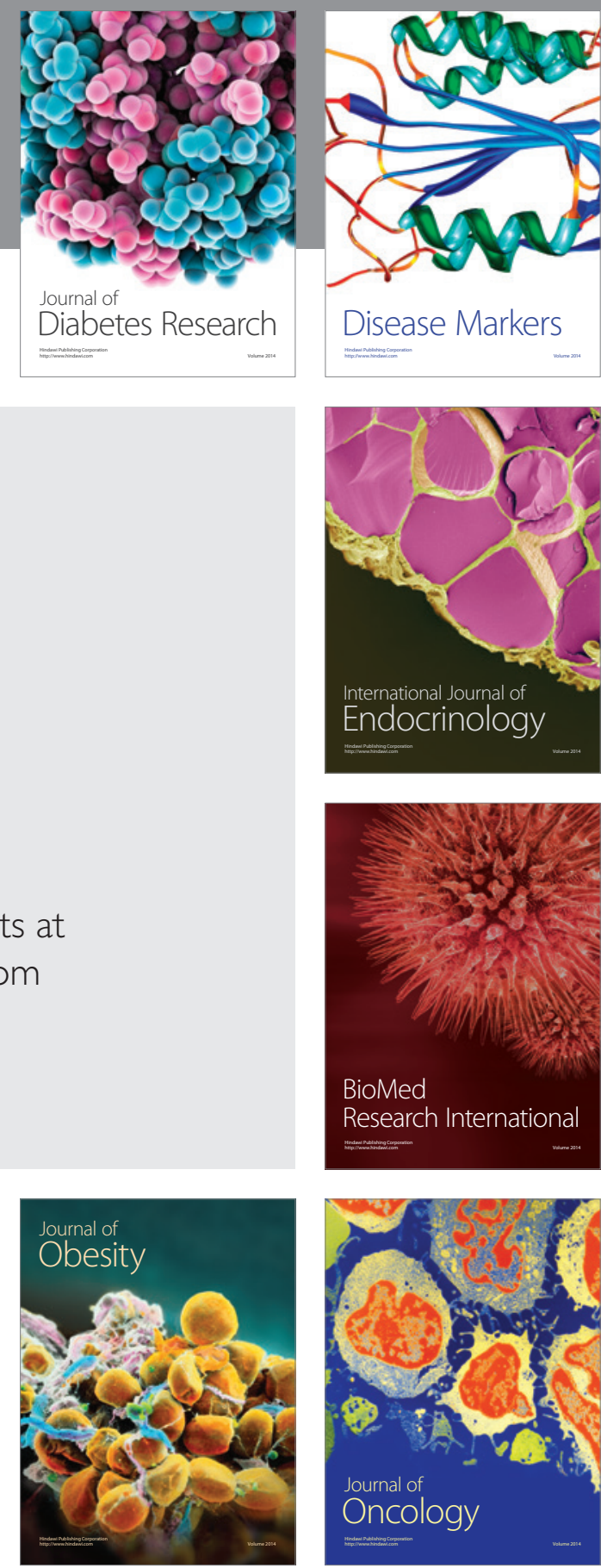

Disease Markers
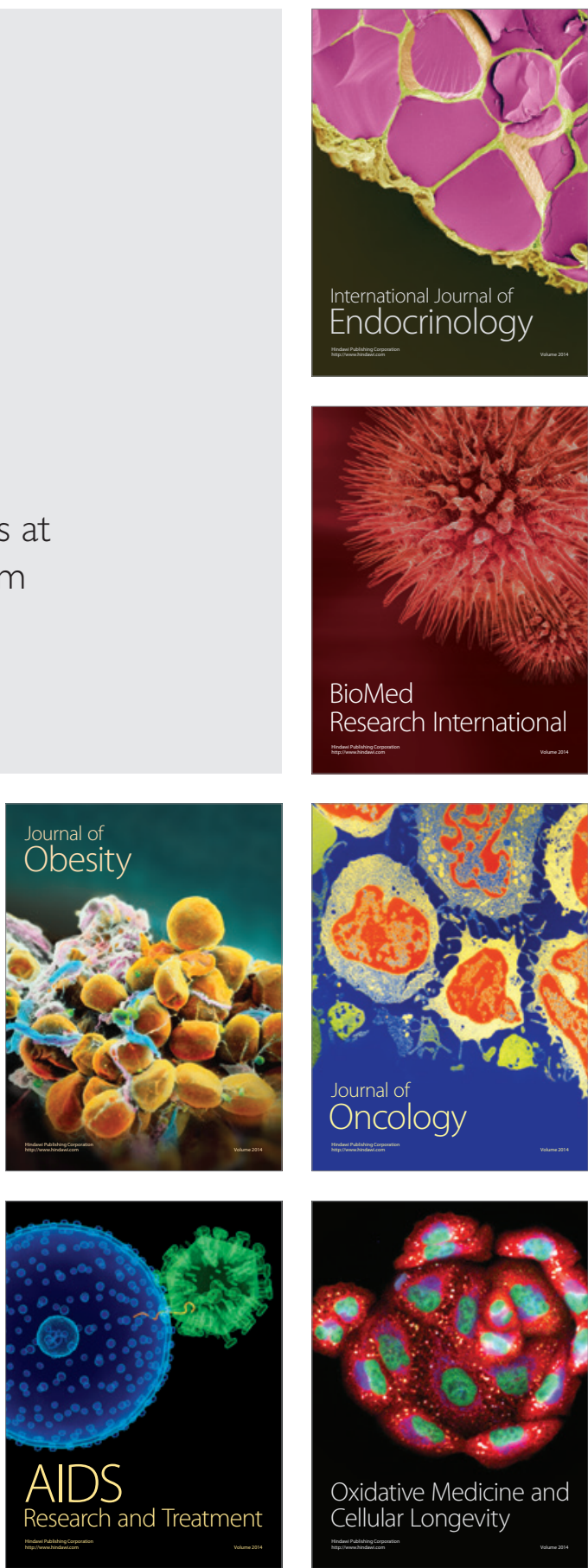